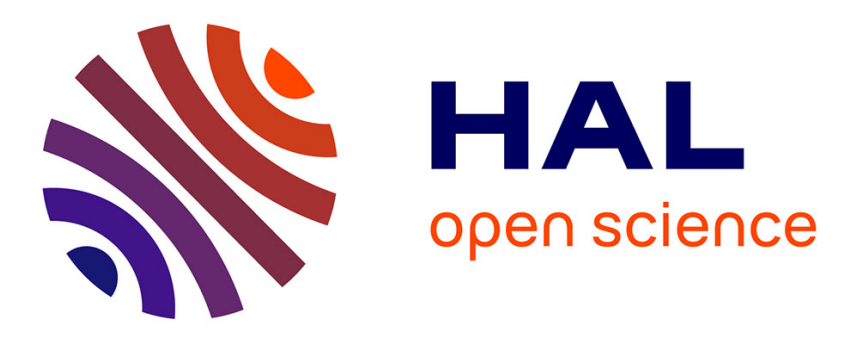

\title{
Numerical simulation of diffusion MRI signals using an adaptive time-stepping method
}

\author{
Jing-Rebecca Li, Donna Calhoun, Cyril Poupon, Denis Le Bihan
}

\section{To cite this version:}

Jing-Rebecca Li, Donna Calhoun, Cyril Poupon, Denis Le Bihan. Numerical simulation of diffusion MRI signals using an adaptive time-stepping method. Physics in Medicine and Biology, 2013, 10.1088/0031-9155/59/2/441 . hal-00763888v2

\section{HAL Id: hal-00763888 \\ https://hal.inria.fr/hal-00763888v2}

Submitted on 7 Jan 2014

HAL is a multi-disciplinary open access archive for the deposit and dissemination of scientific research documents, whether they are published or not. The documents may come from teaching and research institutions in France or abroad, or from public or private research centers.
L'archive ouverte pluridisciplinaire HAL, est destinée au dépôt et à la diffusion de documents scientifiques de niveau recherche, publiés ou non, émanant des établissements d'enseignement et de recherche français ou étrangers, des laboratoires publics ou privés. 


\title{
Numerical simulation of diffusion MRI signals using an adaptive time-stepping method
}

\author{
Jing-Rebecca Li $^{1,3}$, Donna Calhoun ${ }^{2}$, Cyril Poupon ${ }^{3}$ and \\ Denis Le Bihan ${ }^{3}$ \\ ${ }^{1}$ INRIA Saclay-Equipe DEFI,CMAP, Ecole Polytechnique Route de Saclay, \\ 91128, Palaiseau Cedex, France \\ ${ }^{2}$ Department of Mathematics Boise State University 1910 University Dr. Boise, \\ ID 83725-1555 \\ ${ }^{3}$ NeuroSpin, CEA Saclay Center 91191 Gif-sur-Yvette Cedex FRANCE \\ E-mail: jingrebecca.li@inria.fr
}

\begin{abstract}
.
The effect on the MRI signal of water diffusion in biological tissues in the presence of applied magnetic field gradient pulses can be modeled by a multiple compartment Bloch-Torrey partial differential equation. We present a method for the numerical solution of this equation by coupling a standard Cartesian spatial discretization with an adaptive time discretization. The time discretization is done using the explicit Runge-Kutta-Chebyshev method, which is more efficient than the Forward Euler time discretization for diffusive-type problems.

We use this approach to simulate the diffusion MRI signal from the extracylindrical compartment in a tissue model of the brain gray matter consisting of cylindrical and spherical cells and illustrate the effect of cell membrane permeability.
\end{abstract}

\section{Introduction}

Biological tissue is a heterogeneous medium, consisting of cells of various sizes and shapes distributed in the extra-cellular space. The cells are separated from each other and from the extra-cellular compartment by the cell membranes. Diffusion magnetic resonance imaging (dMRI) is an imaging modality that subjects the tissue to various magnetic field gradients and gives a measure of the average displacement of water molecules over a time period on the order of tens of milliseconds.

If the water exchange time between the cells and the extra-cellular space is long compared to the measured diffusion time, then the cell membranes can be approximated as impermeable to water passage. In this case, various analytical and semi-analytical expressions have been obtained for the dMRI signal arising from inside the cells (Crank 1975, Callaghan 1997, Pfeuffer et al. 1998, Sukstanskii \& Yablonskiy 2002). To generalize these results to the complex cellular geometries of biological tissues, geometrical models of the tissue as spherical and oriented cylindrical cells embedded in extra-cellular space were proposed for the brain white matter (Assaf \& Basser 2005, Sen \& Basser 2005) and gray matter (Jespersen et al. 2007). The dMRI signal is then decomposed as the sum of the signals from two different tissue compartments: with the signal from the cylindrical cells being an analytical expression, and the signal from the spherical cells plus the extra-cellular space being Gaussian with an effective diffusion tensor. 
If the cell membranes are permeable, but not so permeable so that the whole tissue can be treated as one compartment, the analysis of the dMRI signal becomes more difficult. If the cells are assumed to be of a special shape: spheres, cubes, or cylinders, and placed on a periodic lattice, then various approximate analytical formulae have been obtained in the long diffusion time limit (Latour et al. 1994, Hasselman \& Johnson 1987, Torquato \& Rintoul 1995). In (Latour et al. 1994, Szafer et al. 1995) approximate analytical formulae for the effective long time diffusion coefficient were applied to the dMRI signal.

Because the analytical results and models of the dMRI signal cited above are subject to certain assumptions such as long diffusion times, it is important to test the various assumptions against a richer numerical model of the dMRI signal that makes less stringent assumptions, as a bridge between analytical models and experimental conditions. Simulation using such numerical models can give new insights that may lead to more accurate analytical models of tissue water diffusion in the future, for use in the estimation of tissue parameters from the experimental signal.

In this paper, we focus on the multiple compartment Bloch-Torrey partial differential equation (PDE), which is a generalization of the Bloch-Torrey PDE (Torrey 1956) to heterogeneous diffusion spatial domains. This PDE models the water proton magnetization subject to diffusion-encoding magnetic field gradient pulses and the dMRI signal is given as the integral of the solution of the PDE at echo time. The numerical solution of the multiple compartment Bloch-Torrey has been considered in the past (Hwang et al. 2003, Xu et al. 2007, Harkins et al. 2009, Russell et al. 2012). The main difference between our approach and those in the previous works is that we use an efficient adaptive time-stepping method, called the Runge-Kutta Chebyshev (RKC) method (Sommeijer et al. 1998), that takes time steps commensurate with the desired accuracy of the time integration at any given point in the simulation. In the case of moderate desired accuracy, we show that this approach is preferred to the explicit Forward Euler method used previously in (Hwang et al. 2003, Xu et al. 2007, Harkins et al. 2009, Russell et al. 2012) where the time step size is limited by numerical stability.

Finally, we use this numerical method to study a particular model of the dMRI signal in the brain gray matter given in (Jespersen et al. 2007), where the neurites are modeled by cylinders. In particular, we examine two assumptions of this model. The first is that the extra-cylindrical compartment, which includes the extra-cellular space and non-cylindrical cells such as glial cells (that we model by spheres), can be considered to undergo Gaussian diffusion. The second is that the signal contribution from the cylinders can be considered to be in 'no-exchange' with the signal contribution from the extra-cylindrical compartment. We study the validity of these assumptions by simulating the dMRI signal at different diffusion times and varying the cell membrane permeabilities.

\section{Theory}

We model the effect on the MRI signal of the water proton magnetization in a biological tissue in the presence of magnetic field gradient pulses by a multiple compartment Bloch-Torrey partial differential equation(Torrey 1956, Price et al. 1998). In the most general case, each compartment corresponds to an individual cell, with an additional compartment being the extra-cellular space (which can be contiguous or not). We can also group all cells of a certain type into a single compartment. We denote the compartments by $\Omega^{l}, l=1,2,3, \cdots$, and their associated intrinsic diffusion coefficients 
by $D^{l}$. If all the compartmental intrinsic diffusion coefficients are equal, then we will refer unambiguously to the intrinsic diffusion coefficient, and denote it with a superscript 0 , as $D^{0}$, where $D^{0}=D^{l}, l=1,2, \cdots$

The union of the compartments $\bigcup_{l=1} \Omega^{l}$ comprises the tissue. Supposing the diffusion-encoding sequence with the time profile $f(t)$, and the diffusion gradient vector $\mathrm{g}$ containing amplitude and direction information of the linear magnetic field gradient, we use the Bloch Torrey PDE model in each compartment: for $\mathbf{r} \in \Omega^{l}$, we have

$\frac{\partial M^{l}(\mathbf{r}, t \mid \mathbf{g})}{\partial t}=-I f(t)(\gamma \mathbf{g} \cdot \mathbf{r}) M^{l}(\mathbf{r}, t \mid \mathbf{g})+\nabla \cdot\left(D^{l} \nabla M^{l}(\mathbf{r}, t \mid \mathbf{g})\right)-\frac{M^{l}(\mathbf{r}, t \mid \mathbf{g})}{\left(T_{2}\right)^{l}}$,

where $\gamma$ is the gyromagnetic ratio of the water proton, $I$ is the imaginary unit, and $\left(T_{2}\right)^{l}$ gives the $T_{2}$ relaxation in $\Omega^{l}$. The magnetization in the entire sample is defined piecewise by the $M^{l}$ 's. The PDE in (1) needs to be supplemented by interface conditions where $\Omega^{l}$ and $\Omega^{n}$ come in contact. We denote the interface between $\Omega^{l}$ and $\Omega^{n}$ by $\Gamma^{l n}$. One interface condition is the continuity of flux:

$$
D^{l}\left(\nabla M^{l}(\mathbf{a}, t \mid \mathbf{g}) \cdot \mathbf{n}^{l}(\mathbf{a})\right)=-D^{n}\left(\nabla M^{n}(\mathbf{a}, t \mid \mathbf{g}) \cdot \mathbf{n}^{n}(\mathbf{a})\right), \mathbf{a} \in \Gamma^{l n},
$$

where $\mathbf{n}^{l}(\mathbf{a})$ and $\mathbf{n}^{n}(\mathbf{a})$ are the outward-point normals to $\Omega^{l}$ and $\Omega^{n}$ at $\mathbf{a}$, so in fact $\mathbf{n}^{l}(\mathbf{a})=-\mathbf{n}^{n}(\mathbf{a})$. This ensures the conservation of magnetization. The second interface condition is:

$$
D^{l}\left(\nabla M^{l}(\mathbf{a}, t \mid \mathbf{g}) \cdot \mathbf{n}^{l}(\mathbf{a})\right)=\kappa^{l n}\left(M^{l}(\mathbf{a}, t \mid \mathbf{g})-M^{n}(\mathbf{a}, t \mid \mathbf{g})\right), \mathbf{a} \in \Gamma^{l n} \cdot(3)
$$

This incorporates a permeability coefficient $\kappa^{l n}$ across $\Gamma^{l n}$ which models the ease with which water crosses the interface. The larger the $\kappa^{l n}$, the easier the passage of water.

For a Pulsed-Gradient Spin Echo (PGSE) (Stejskal \& Tanner 1965) sequence, made of two rectangular pulses (duration $\delta$, separated by a time interval $\Delta-\delta$ ) the profile $f(t)$ is:

$$
f(t)= \begin{cases}1, & t_{1} \leq t \leq t_{1}+\delta, \\ -1, & t_{1}+\Delta<t \leq t_{1}+\Delta+\delta, \\ 0, & \text { elsewhere }\end{cases}
$$

where $t_{1}$ is the start of the first pulse with $t_{1}+\Delta>T E / 2$, TE is the echo time when the signal is measured.

The PDE and interface conditions in $(1,2,3)$ must be supplemented with an initial condition:

$$
M(\mathbf{r}, 0 \mid \mathbf{g})=\rho^{l}, \mathbf{r} \in \Omega^{l}, \text { for } l=1,2, \cdots,
$$

where $\rho^{l}$ is the water density in $\Omega^{l}$. The total signal attenuation is:

$$
S(\mathbf{g}, t):=\frac{\sum_{l} \int_{\mathbf{r} \in \Omega^{l}} M^{l}(\mathbf{r}, t \mid \mathbf{g}) d \mathbf{r}}{\sum_{l} \rho^{l} V^{l}},
$$

where $V^{l}$ is the volume of $\Omega^{l}$. In the case of a compartment $\Omega^{l}$ that does not exchange water with the domain outside of $\Omega^{l}$, if we want to study the contribution to the dMRI signal from this compartment, we define the partial signal attenuation due to $\Omega^{l}$ :

$$
S^{l}(\mathbf{g}, t):=\frac{\int_{\mathbf{r} \in \Omega^{l}} M^{l}(\mathbf{r}, t \mid \mathbf{g}) d \mathbf{r}}{\rho^{l} V^{l}},
$$

which will be useful to study the contribution to the total signal from the different physical compartments. The dMRI signal is measured at echo time $t=T E \geq \Delta+\delta$. 
In a homogeneous medium, the signal attenuation is $e^{-D^{0} b}$, where $D^{0}$ is the intrinsic diffusion coefficient, and the b-value is a weighting factor that combines the effects of the imaging and diffusion gradients (Le Bihan et al. 1986) and which is:

$$
b(\mathbf{g}, \delta, \Delta)=\gamma^{2}\|\mathbf{g}\|^{2} \delta^{2}(\Delta-\delta / 3)
$$

for the PGSE sequence(Stejskal \& Tanner 1965). In biological tissue, the signal attenuation is not a simple decaying exponential in $b$ and the slope of $\log S(b)$ at $b=0$ is not simply related to the intrinsic diffusion coefficients of the physical compartments. Theoretical analysis of the signal attenuation as a function of the b-value is difficult for general gradient pulses and is usually done only in the narrow pulse limit. Let $u\left(\mathbf{r}, t, \mid \mathbf{r}_{0}\right)$ be the probability of finding water molecules originally at $\mathbf{r}_{0}(t=0)$ in position $\mathbf{r}$ at time $t$, then the signal in the narrow pulse (NP) limit is:

$$
S^{N P}(\|\mathbf{g}\| \delta \gamma, \Delta)=\int_{\mathbf{r}_{0} \in \mathbb{R}^{3}} \int_{\mathbf{r} \in \mathbb{R}^{3}} e^{-I\|\mathbf{g}\| \delta \gamma\left(\mathbf{r}-\mathbf{r}_{0}\right) \cdot \mathbf{u}_{\mathbf{g}}} u\left(\mathbf{r}, \Delta, \mid \mathbf{r}_{0}\right) d \mathbf{r} d \mathbf{r}_{0},(9)
$$

where $\mathbf{u}_{\mathbf{g}}:=\mathbf{g} /\|\mathbf{g}\|$ is the normalized gradient direction. We chose the independent variable in $S^{N P}$ to be $\|\mathbf{g}\| \delta \gamma$ because the interior integral over $\mathbf{r}$ in (9) is just a one dimensional Fourier transform, with the Fourier variable being $\|\mathbf{g}\| \delta \gamma$ and the spatial variable being $\left(\mathbf{r}-\mathbf{r}_{0}\right) \cdot \mathbf{u}_{\mathbf{g}}$, leading us to the property relating derivatives of the Fourier transform and the moments of the original function:

$I^{2 n} \frac{d^{2 n} S^{N P}}{d(\|\mathbf{g}\| \delta \gamma)^{2 n}}(\|\mathbf{g}\| \delta \gamma, \Delta)=\int_{\mathbf{r}_{0} \in \mathbb{R}^{3}} \int_{\mathbf{r} \in \mathbb{R}^{3}} e^{-I \mathbf{g} \delta \gamma\left(\mathbf{r}-\mathbf{r}_{0}\right)}\left(\left(\mathbf{r}-\mathbf{r}_{0}\right) \cdot \mathbf{u}_{\mathbf{g}}\right)^{2 n} u\left(\mathbf{r}, \Delta \mid \mathbf{r}_{0}\right) d \mathbf{r} d \mathbf{r}_{0}$.

In a dMRI experiment, the pulse sequence profile $f(t)$ is most often fixed while $\mathbf{g}$ is varied in amplitude, so we can treat $\Delta$ as a constant and use the new independent variable $b=\gamma^{2}\|\mathbf{g}\|^{2} \delta^{2} \Delta$ to obtain for $S^{N P}(b)$ :

$$
\frac{d^{n} S^{N P}}{d b^{n}}(0, \Delta)=\frac{(-1)^{n} n !}{(2 n) !} \frac{1}{\Delta^{n}} \int_{\mathbf{r}_{0} \in \mathbb{R}^{3}} \int_{\mathbf{r} \in \mathbb{R}^{3}}\left(\left(\mathbf{r}-\mathbf{r}_{0}\right) \cdot \mathbf{u}_{\mathbf{g}}\right)^{2 n} u\left(\mathbf{r}, \Delta \mid \mathbf{r}_{0}\right) d \mathbf{r} d \mathbf{r}_{0} .
$$

Thus in the narrow pulse limit, the analytical derivatives of $S$ give the moments of the probability density function $u\left(\mathbf{r}, t, \mid \mathbf{r}_{0}\right)$, averaged over all starting positions $\mathbf{r}_{0}$. For general pulse sequences, (10) is not exact.

The first and second analytical derivatives of $S$ can be related to the Apparent Diffusion Coefficient and the Apparent Kurtosis(Chabert et al. 2005, Jensen et al. 2005, Frohlich et al. 2006) computed in dMRI:

$$
\begin{aligned}
& A D C_{0}=-\frac{d \log S}{d b}(0)=-\frac{d S}{d b}(0), \\
& A K_{0}=3 \frac{d^{2} \log S}{d b^{2}}(0)\left(\frac{d \log S}{d b}(0)\right)^{-2}=3 \frac{d^{2} S}{d b^{2}}(0)\left(\frac{d S}{d b}(0)\right)^{-2}-3,
\end{aligned}
$$

where we denoted the quantities by $A D C_{0}$ and $A K_{0}$, respectively, to emphasize that the analytical derivatives of $\log S(b)$ are taken at $b=0$. The $A D C_{0}$ and the $A K_{0}$ are interesting because the $A D C_{0}$ gives an indication of the mean squared distance traveled by water molecules, averaged over all the compartments, and $A K_{0}$ gives an indication of the deviation from Gaussian diffusion. We computed $A D C_{0}$ and $A K_{0}$ by fitting $\log S(b)$ at a set of $b$ by polynomials in a least squares sense. We start with a polynomial of degree 1 (where the second derivative is set to 0 ) and increase the polynomial degree until the $A D C_{0}$ of the polynomial fit of degree $n$ and the $A D C_{0}$ the fit of degree $n+1$ are within 1 percent (relative error) of each other and for $A K_{0}$ we allow 5 percent relative error. 
Numerical simulation of diffusion MRI signals

\section{Method}

Our approach solves the multiple compartment Bloch-Torrey PDE in three dimensions by coupling a standard Cartesian spatial discretization that allows permeable membranes to the Runge-Kutta-Chebyshev (RKC) method (Sommeijer et al. 1998), which is an adaptive time-stepping method that enforces a user-specified time integration error tolerance at each spatial discretization point.

\subsection{Cartesian spatial discretization}

In the spatial discretization, we use a rectangular computational domain $C=[0, L] \times$ $[0, L] \times[0, L]$ that contains a configuration of cells and a Cartesian discretization of $C$. We use the same transformation on the Bloch-Torrey PDE as (Russell et al. 2012), where a change of variable is made so that the unknown function to be solved becomes

$$
m(\mathbf{r}, t \mid \mathbf{g}):=M(\mathbf{r}, t \mid \mathbf{g}) e^{I \gamma \mathbf{g} \cdot \mathbf{r} \int_{0}^{t} f(s) d s} .
$$

We include here the interface condition at the intersection of two different compartments because this was not explicitly described in (Russell et al. 2012). We denote by $\left\{x_{1}, \cdots x_{W}\right\}$ and $\left\{y_{1}, \cdots, y_{W}\right\}$ and $\left\{z_{1}, \cdots, z_{W}\right\}$ the discretization points in the $x, y, z$ coordinates, respectively:

$$
x_{i}=i h-h / 2, \quad y_{j}=j h-h / 2, \quad z_{k}=k h-h / 2,
$$

where the $h$ is the side length of one discretization element. The diffusion coefficient at $\mathbf{r}_{i j k} \equiv\left(x_{i}, y_{j}, z_{k}\right)$ will be denoted by $D_{i j k}$. We define $\mathbf{g}:=\left(g_{x}, g_{y}, g_{z}\right)$ and

$$
\mathbf{A}:=\left(A_{x}, A_{y}, A_{z}\right):=\left(g_{x}, g_{y}, g_{z}\right) \gamma \int_{0}^{t} f(s) d s .
$$

The discretization formula for $m$ is:

$$
\begin{aligned}
& \frac{\partial m_{i j k}(t)}{\partial t}=-\frac{m_{i j k}(t)}{\left(T_{2}\right)_{i j k}}+\frac{1}{h^{2}} \\
& \left(\left(D_{i+\frac{1}{2}, j k}^{*}\left(e^{-I A_{x} h} m_{i+1, j, k}(t)-m_{i j k}(t)\right)-D_{i-\frac{1}{2}, j k}^{*}\left(m_{i j k}(t)-e^{I A_{x} h} m_{i-1, j, k}(t)\right)\right)\right. \\
& +\left(D_{i, j+\frac{1}{2}, k}^{*}\left(e^{-I A_{y} h} m_{i, j+1, k}(t)-m_{i j k}(t)\right)-D_{i, j-\frac{1}{2}, k}^{*}\left(m_{i j k}(t)-e^{I A_{y} h} m_{i, j-1, k}(t)\right)\right) \\
& \left.+\left(D_{i j, k+\frac{1}{2}}^{*}\left(e^{-I A_{z} h} m_{i j, k+1}(t)-m_{i j k}(t)\right)-D_{i j, k-\frac{1}{2}}^{*}\left(m_{i j k}(t)-e^{I A_{z} h} m_{i j, k-1}(t)\right)\right)\right) .
\end{aligned}
$$

If the diffusion coefficient is continuous in $x$ at $\left(x_{i+\frac{1}{2}}, y_{j}, z_{k}\right)$, then $D_{i+\frac{1}{2}, j k}^{*}=D_{i j k}$. Otherwise, $D^{*}$ is given by

$$
D_{i+\frac{1}{2}, j k}^{*}=\frac{1}{\left(\frac{1}{2}\left(\frac{1}{D\left(x_{i+1}\right)}+\frac{1}{D\left(x_{i}\right)}\right)+\frac{1}{\kappa^{i, i+1} h}\right)} .
$$

Similarly in the other coordinate directions.

Same as (Xu et al. 2007, Russell et al. 2012), we assume the computational domain $C$ is extended by periodic copies of itself to handle the diffusion of water molecules close to the boundary of $C$. The boundary conditions on $m$ are periodic on the faces of $C$, as was shown in (Russell et al. 2012). The spatial discretization in (14) gives rise to a system of ODEs in time which we will solve by a time stepping method described in the next section. 


\subsection{RKC time stepping method}

We solve the system of ODEs in (14) using the Runge-Kutta-Chebyshev (RKC) method (Sommeijer et al. 1998). We briefly describe this method as it applies to the solution of

$$
\frac{d\left\{m_{i j k}(t)\right\}}{d t}=F\left(t,\left\{m_{i j k}(t)\right\}\right),
$$

where $F$ is the discrete Laplacian plus the $T_{2}$ decay term defined in the right hand side of (14). The details of the RKC method can be found in the paper referenced above. To go from $\left\{m_{i j k}\right\}^{n} \approx\left\{m_{i j k}\left(t^{n}\right)\right\}$ to $\left\{m_{i j k}\right\}^{n+1} \approx\left\{m_{i j k}\left(t^{n+1}\right)\right\}$, the following $s$ stages are taken:

$$
\begin{aligned}
Y^{0} & =\left\{m_{i j k}\right\}^{n}, \\
Y^{1} & =Y^{0}+\mu^{1} \tau F\left(t^{n},\left\{m_{i j k}\right\}^{n}\right), \\
Y^{j} & =\mu^{j} Y^{j-1}+\nu^{j} Y^{j-2}+\left(1-\mu^{j}-\nu^{j}\right) Y^{0}+\tilde{\mu} \tau F\left(t^{n}+c^{j-1} \tau, Y^{j-1}\right) \\
& +\tilde{\gamma} \tau F\left(t^{n}, Y^{0}\right), \quad 2 \leq j \leq s, \\
\left\{m_{i j k}\right\}^{n+1} & =Y^{s},
\end{aligned}
$$

where $\tau=\left(t^{n+1}-t^{n}\right)$ and the coefficients are determined by Chebyshev polynomials.

The two important things to note about the RKC method are that the local time error at $t^{n+1}$ can be estimated as:

$e r r^{n+1}=\frac{1}{15}\left(12\left(\left\{m_{i j k}\right\}^{n}-\left\{m_{i j k}\right\}^{n+1}\right)+6 \tau\left(F\left(t^{n},\left\{m_{i j k}\right\}^{n}\right)+F\left(t^{n+1},\left\{m_{i j k}\right\}^{n+1}\right)\right)\right)$,

and that the time step can be made stable no matter how large it is by increasing the number of stages $s$. Specifically, the stability criterion is (Sommeijer et al. 1998):

$\left(t^{n+1}-t^{n}\right)_{R K C} \leq \Delta t_{R K C}^{\max }:=\frac{0.653 s^{2}}{\max \operatorname{eig}\left(j a c F\left(t,\left\{m_{i j k}\right\}(t)\right)\right)} \approx 0.653 s^{2} \frac{h^{2}}{6 D^{0}}$.

Essentially, the RKC method chooses a $t^{n+1}$ where the error term above is smaller than the user-specified tolerance and the number of stages $s$ is increased so that $t^{n+1}-t^{n}$ is a stable step. Because the time step can be enlarged as $O\left(s^{2}\right)$ whereas the computational time only increases as $O(s)$, this means that the RKC method with larger time steps computes the solution faster than taking many smaller steps of the Forward Euler method. The number of stages $s$ is typically between 30-50 in the simulations we have performed. In addition, because of the existence of a threeterm recurrence relation for Chebyshev polynomials. the storage requirement does not increase with $s$, it stays constant at 5 times the number of unknowns.

In contrast, for the Forward Euler method, the stability criterion for a spatial discretization $h$ is

$$
\left(t^{n+1}-t^{n}\right)_{F E} \leq \Delta t_{F E}^{\max }:=\frac{h^{2}}{6 D^{0}} .
$$

For moderate accuracy requirements, it is usually much more efficient to increase $s$ according to (16) to get a time step that is appropriate for the desired accuracy than being limited by the stability condition of (17).

Because it is difficult to judge the computational time which depends on the computer on which the simulation was performed, we define a quantity called 
the computational efficiency of the RKC method over the Forward Euler method, $E_{R K C}\left(t^{n}\right)$ at the RKC time point $t^{n}$, in the following way:

$$
E_{R K C}\left(t^{n}\right)=\frac{\left(t^{n+1}-t^{n}\right) / P_{R K C}\left(t^{n}, t^{n+1}\right)}{\Delta t_{F E}^{\max }},
$$

where $P_{R K C}\left(t^{n}, t^{n+1}\right)$ is the number of Laplacian evaluations in advancing from $t^{n}$ to the next $t^{n+1}$ using the RKC method. The maximum time step of the Forward Euler method $\Delta t_{F E}^{\max }$ is given in (17). The number of Laplacian evaluations in advancing from $t$ to $t+\Delta t$ using the Forward Euler method is 1 . The quantity $E_{R K C}\left(t^{n}\right)$ measures how much faster the RKC method runs compared to the Forward Euler method on the interval $\left[t^{n}, t^{n+1}\right]$.

\section{Numerical results and discussion}

We implemented the above method as a Fortran90 program. The implementation of the RKC method comes from the publicly available Fortran code downloaded from http://www.netlib.org/ode/rkc.f. Our simulations were performed on a Dell network server (Intel Xeon E5-2667 processor 2.90GHz), running the program as a serial code.

\subsection{A dMRI signal model for brain gray matter}

In the brain gray matter, neurons, consisting of a large neuronal body and long extensions (axons and dendrites), are densely packed. There are also glial cells which do not have these long extensions. The extra-cellular space accounts for only a small fraction of the total volume. In (Jespersen et al. 2007), a simplified model of the dMRI signal was proposed:

$$
S(b)=\left(1-v^{c}\right) e^{-D^{e c} b}+v^{c} S^{c}(b),
$$

where $v^{c}$ is the volume fraction of all the diffusion compartments exhibiting cylindrical symmetry and everything outside of these cylindrical compartments is assumed to undergo Gaussian diffusion with an effective diffusion coefficient $D^{e c}$, where the superscript "ec" stands for extra-cylindrical. The term $S^{c}(b)$ is the signal due to the cylindrical compartments under the assumption that they are impermeable with respect to the extra-cylindrical compartment. In the gray matter, where there is no a-priori orientation preference for the neurites (axons and dendrites), the cylindrical compartments consist of the neurites themselves. We are interested in simulating the dMRI signal from regions of the brain where the neurites can be thought of as oriented more or less randomly.

\subsection{DMRI signal from the extra-cylindrical compartment}

First we study the dMRI signal from the compartment consisting of the extracellular space and the spherical cells. We begin by constructing a geometry consisting of generally oriented cylinders and spheres. In the computational box $C=[-12.5 \mu m, 12.5 \mu m]^{3}$, we placed $N^{c}=250$ randomly-placed points (uniformly distributed in $C$ ). At each point, we extended an infinite cylinder of radius $R^{c}$ oriented in a random direction (drawn uniformly from the unit sphere) and cut the cylinder off at the boundaries of $C$. The size of $C$ is chosen for the simulation of the extracylindrical space, where we observed that the effective diffusion coefficient was no more than $10^{-3} \mathrm{~mm}^{2} / \mathrm{s}$, meaning a diffusion distance of $15 \mu \mathrm{m}$ in $40 \mathrm{~ms}$. This size ensures 
that most molecules see $C$ no more than twice during $40 \mathrm{~ms}$. The cylindrical volume fraction will be denoted $v^{c}$. We also distribute $N^{s}=10$ randomly-placed spheres of radius $R^{s}$ in $C$ and denote the spherical cells volume fraction by $v^{s}$. The volume fraction of the extra-cellular space will be denoted $v^{e}=1-v^{c}-v^{s}$. See Fig 1(a) for a rendering of the geometry with $R^{c}=1.25 \mu \mathrm{m}, R^{s}=4 \mu \mathrm{m}, v^{e}=0.15$ and $v^{s}=0.15$. We discretized $C$ by a regular mesh with spatial spacing $h$. See Fig 1(b) for a cross section view of the discretized mesh at $h=0.125 \mu \mathrm{m}$.

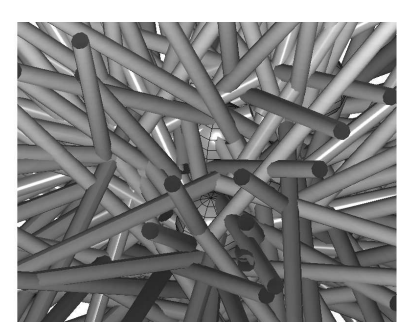

(a) Geometry

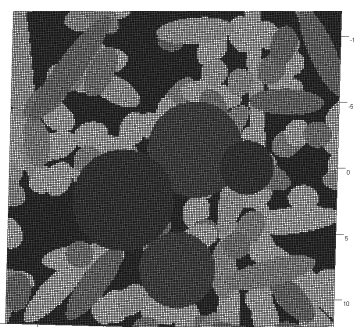

(b) Cross section of mesh

Figure 1. Left: a rendering of the simulation geometry consisting of 250 randomly placed and oriented cylinders and 10 randomly placed spheres (zoomedin view). Right: a cross section view of the discretized mesh of the geometry with $h=0.125 \mu m$ (zoomed-in view).

At the start of the simulation, we placed water molecules uniformly in the spherical cells and the extra-cellular space and placed no water molecules inside the cylinders. We made the cylindrical cells impermeable so that the water molecules are blocked from entering the cylinders during the simulation. We varied the spherical cell permeability: $\kappa^{s}=$ $0 \mathrm{~m} / \mathrm{s}$ (impermeable), $10^{-5} \mathrm{~m} / \mathrm{s}, 10^{-4} \mathrm{~m} / \mathrm{s}, \infty$ (infinitely permeable), and we computed the $A D C_{0}$ and $A K_{0}$ from b-values: 0, 250, 500,750, 1000, 1250, 1500,1750, 2000s/ $\mathrm{mm}^{2}$. The RKC tolerance was set to tol $=10^{-4}$, after doing a preliminary simulation for free diffusion where we saw that the relative signal error was less than 0.005 at $b=2000 \mathrm{~s} / \mathrm{mm}^{2}$, which is sufficiently accurate for the dMRI application where there is significant experimental noise in the measured signal.

We chose a constant intrinsic diffusion coefficient $D^{0}=3 \times 10^{-3} \mathrm{~mm}^{2} / \mathrm{s}$ in the cells and the extra-cellular space and neglected the $T_{2}$ relaxation term. The diffusion-encoding gradient sequence simulated was PGSE, $\delta=2.5 \mathrm{~ms}$ and we varied $\Delta=10 \mathrm{~ms}, 20 \mathrm{~ms}, 30 \mathrm{~ms}, 40 \mathrm{~ms}$. We simulated the dMRI signal on two meshes with $h=0.25 \mu \mathrm{m}$ and $h=0.125 \mu \mathrm{m}$.

We examine the results for the mesh with $h=0.125 \mu m$ (marked by stars). In Fig. 2, we see that at $\kappa^{s}=0 \mathrm{~m} / \mathrm{s}$ (solid line) and $\kappa^{s}=10^{-5} \mathrm{~m} / \mathrm{s}$ (dash-dots), the $A D C_{0}$ decreases from $0.4 \times 10^{-3} \mathrm{~mm}^{2} / \mathrm{s}$ to $0.2 \times 10^{-3} \mathrm{~mm}^{2} / \mathrm{s}$ as the diffusion time is increased from $10 \mathrm{~ms}$ to $40 \mathrm{~ms}$, while the $A K_{0}$ goes from 1.8 to 2.7 for $\kappa^{s}=10^{-5} \mathrm{~m} / \mathrm{s}$ and from 2 to 3.5 for $\kappa^{s}=0 \mathrm{~m} / \mathrm{s}$. At $\kappa^{s}=10^{-4} \mathrm{~m} / \mathrm{s}$ (dashes), the $A D C_{0}$ decreases from $0.55 \times 10^{-3} \mathrm{~mm}^{2} / \mathrm{s}$ to $0.45 \times 10^{-3} \mathrm{~mm}^{2} / \mathrm{s}$, while the $A K_{0}$ stays around 1.2 . At $\kappa^{s}=\infty \mathrm{m} / \mathrm{s}$ (dots), the $A D C_{0}$ decreases from $1.05 \times 10^{-3} \mathrm{~mm}^{2} / \mathrm{s}$ to $0.85 \times 10^{-3} \mathrm{~mm}^{2} / \mathrm{s}$, while the $A K_{0}$ stays around 0.6. Thus we see that the $A K_{0}$ is quite high between $10 \mathrm{~ms}$ and $40 \mathrm{~ms}$ for the different values of the spherical cells permeability. Because the $A K_{0}$ is so high at finite permeability, this set of simulations put to question the assumption 
that the extra-cellular space and the spherical cells can be considered as one physical compartment experiencing Gaussian diffusion at the above diffusion times when the b-values are as high as $2000 \mathrm{~s} / \mathrm{mm}^{2}$.

From the same Figure, we also see that when we use a coarser mesh with $h=0.25 \mu m$ (marked by circles), the values of $A D C_{0}$ and $A K_{0}$ are slightly shifted, with the $A D C_{0}$ being lower at the coarser discretization, which is easily explained by the cylinders having more 'jagged' surfaces. However, the behavior of the $A D C_{0}$ and $A K_{0}$ as a function of $\kappa^{s}$ and diffusion time is consistent with the results from the finer mesh.

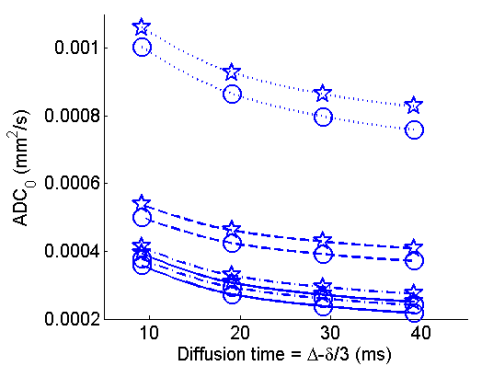

(a) $A D C_{0}$

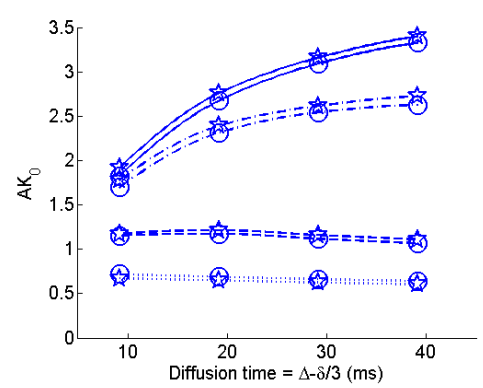

(b) $A K_{0}$

Figure 2. In the extra-cylindrical compartment comprising of the spherical cells $\left(v^{s}=0.15\right)$ and the extra-cellular space $\left(v^{e}=0.15\right)$ and where the water molecules are stopped from entering the cylindrical cells, the $A D C_{0}$ and the $A K_{0}$ are computed for the signal arising only from this compartment. Four values of spherical cell permeability: $\kappa^{s}=0 \mathrm{~m} / \mathrm{s}$ (solid line), $\kappa=1 \times 10^{-5} \mathrm{~m} / \mathrm{s}$ (dash-dots), $\kappa=1 \times 10^{-4} \mathrm{~m} / \mathrm{s}$ (dashes), $\kappa=\infty \mathrm{m} / \mathrm{s}$ (dots), were simulated on two meshes with spatial discretization $h=0.25 \mu \mathrm{m}$ (circles) and $h=0.125 \mu \mathrm{m}$ (stars).

To study the effect of $R^{c}$ and $R^{s}$ on the dMRI signal, we set $\kappa^{s}=0$ and computed the $A D C_{0}$ and $A K_{0}$ in the extra-cellular and the spherical compartments separately. Making $\kappa^{s}=0$ means we do not have to account for the exchange between them and can focus on the shape of the compartments. We fixed the positions of the cylinders and spheres as the previous example and simulated three cases:

(i) $R^{c}=1 \mu m, R^{s}=4 \mu m, v^{e}=0.28, v^{s}=0.15$ (circles in Fig 3),

(ii) $R^{c}=1 \mu m, R^{s}=5 \mu m, v^{e}=0.25, v^{s}=0.25$ (crosses in Fig 3),

(iii) $R^{c}=1.25 \mu m, R^{s}=4 \mu m, v^{e}=0.15, v^{s}=0.15$ (stars in Fig 3).

In Fig 3 we show the $A D C_{0}$ of the extra-cellular compartment (EX), the spheres compartment $(\mathrm{SPH})$, and the combined extra-cylindrical compartment $(\mathrm{EX}+\mathrm{SPH})$. We see that it is the extra-cellular space that dominates the $A D C_{0}$ of the combined compartment in the range of diffusion times $10-40 \mathrm{~ms}$. When the spheres are larger, even though the diffusion inside of the spheres is more significant, it does not compensate for the decreased diffusion in the extra-cellular space. Thus, to study the cell features beyond the extra-cellular space, one must look beyond the $A D C_{0}$ and to information contained in the higher b-values. 


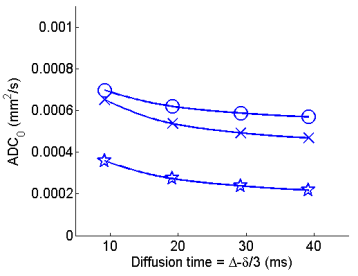

(a) $A D C_{0}(E X+S P H)$

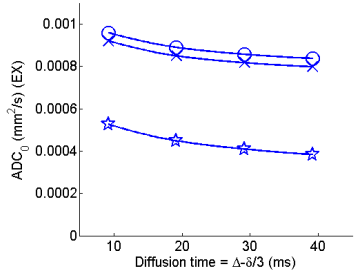

(b) $A D C_{0}(E X)$

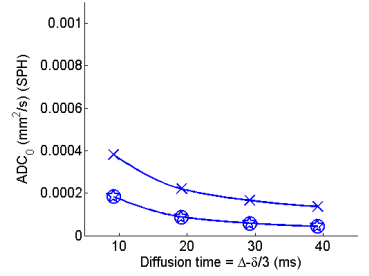

(c) $A D C_{0}(S P H)$

Figure 3. Fixing the positions of the cylinders and spheres as in Fig 2 and setting $\kappa^{s}=0$ to study the $A D C_{0}$ of the spherical cells and the extra-cellular space separately as $R^{c}$ and $R^{s}$ are varied.

Legend: Circles: $R^{c}=1 \mu m, R^{s}=4 \mu m\left(v^{e}=0.28, v^{s}=0.15\right)$. Crosses: $R^{c}=1 \mu m, R^{s}=5 \mu m\left(v^{e}=0.25, v^{s}=0.25\right)$. Stars: $R^{c}=1.25 \mu m, R^{s}=4 \mu m$ $\left(v^{e}=0.15, v^{s}=0.15\right)$.

\subsection{Performance of the numerical method}

We show the efficiency of the RKC time-stepping method over the Forward Euler method for the example shown in Fig. 2 for the PGSE sequence, $\delta=5 \mathrm{~ms}, \Delta=40 \mathrm{~ms}$, at $b=1000 \mathrm{~s} / \mathrm{mm}^{2}$, using the two spatial meshes: $h=0.25 \mu \mathrm{m}$ and $h=0.125 \mu \mathrm{m}$. The efficiency at a RKC time step $\left[t_{n}, t_{n+1}\right]$ is defined as the ratio of the number of Laplacian evaluations needed by the RKC method to advance the solution from $t_{n}$ to $t_{n+1}$ to the number of Laplacian evaluations required by the Forward Euler method to advance from $t_{n}$ to $t_{n+1}$. We see in Fig 4(a) that during the time the gradient is turned off: $2.5 \mathrm{~ms} \leq t \leq 40 \mathrm{~ms}$, the efficiency of the RKC method varies from 1 to 90 , meaning, as a rough estimate, the RKC method is about 45 times faster than the Forward Euler method during the full simulation. When $h=0.125 \mu \mathrm{m}$, efficiency of the RKC method varies from 1 to 40, meaning a rough estimate that RKC is 20 times faster than the Forward Euler method during the full simulation. The reason for the difference is that on a coarse spatial mesh, the solution error cannot be reduced by time refinement. In this case, RKC takes very large time steps, with a large number of stages. When $h$ is smaller, then the time discretization error can be made lower by making the time steps smaller. In this case, RKC takes small time steps. Reassuringly, we see that RKC takes small steps when the gradient suddenly changes value: at $t=0,2.5 \mathrm{~ms}$ and $t=40 \mathrm{~ms}$, because the time refinement is needed there. Thus, we see that in general the RKC method is much faster than the Forward Euler method for diffusion problems where the time accuracy requirement is much less restrictive than the numerical stability requirement for Forward Euler. Also, the RKC time steps are completely adapted to the problem to be solved, refining where the gradient suddenly changes. The computational time on the mesh $h=0.25 \mu \mathrm{m}\left(100^{3}\right.$ unknowns $)$ is around 2 minutes per b-value and it is 30 minutes per b-value on the mesh $h=0.125 \mu \mathrm{m}\left(200^{3}\right.$ unknowns) on a Dell network server (Intel Xeon E5-2667 processor 2.90GHz), while running the program as a serial code.

To simulate the total tissue with cylindrical cells in water exchange with the the extra-cylindrical compartment, it would be indispensable to create a geometry of densely packed thin cylinders, where the cylinders do not cross each other. We were not able to define such a geometry because straight cylinders cross each other 


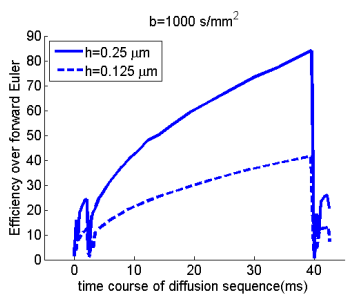

(a) Efficiency of RKC

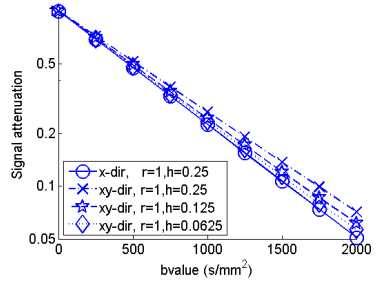

(b) Signal at $\Delta=10 \mathrm{~ms}$

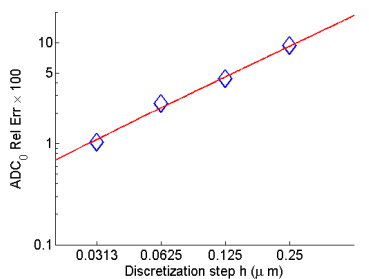

(c) $A D C_{0}$ converges in $O\left(h^{1}\right)$

Figure 4. Left: Efficiency of RKC over the Forward Euler time stepping method for two discretizations of the extra-cylindrical compartment whose results are shown in Fig 2. The efficiency of the RKC method is how much faster the RKC method runs than the Forward Euler method during the course of simulation $\left(=E_{R K C}\right.$ defined in (18)). Center and right: study of numerical error associated with slanted cylinders when using a Cartesian discretization mesh. DMRI signals were computed by putting water molecules only inside a single impermeable cylinder oriented 45 degrees with respect to the diffusion gradient direction. " $\mathrm{x}$ dir": cylinder oriented parallel to $[1,0,0]$. "xy-dir": cylinder oriented parallel to $[1,1,0]$. Center: For both cylinder orientations, the $\log S(b)$ are straight lines in $b$, but with slightly differing slopes. The theoretical value of $A D C_{0}$ should be $A D C_{0}^{c y l}=D^{0} / 2=1.5 \times 10^{-3} \mathrm{~mm}^{2} / \mathrm{s}$. Right: $A D C_{0}$ relative error in percent= $\left(A D C_{0}-A D C_{0}^{c y l}\right) / A D C_{0}^{c y l} \times 100$ for "xy-dir" cylinders at $\Delta=10 \mathrm{~ms}$. The line connecting the four data points is the linear fit of the logarithm of $h$ to the logarithm of the $A D C_{0}$ error.

at many places if there are a lot of them in a small volume. This problem of defining non-crossing cylinders was not important in the previous section where we simulated the diffusion in the extra-cylindrical compartment because we only needed the space outside of the cylinders. However, this problem prevents us from simulating the complete problem of diffusion in a tissue model containing densely packed, randomly oriented, permeable cylindrical cells.

Instead, here we simply study the discretization errors associated with the 'jagged surface' of a slanted cylinder due to a Cartesian mesh when simulating diffusion inside this cylinder. This may be useful in the future when the problem of non-crossing cylinders placement has been solved and the simulation of the full tissue model can be done. For a thin straight impermeable cylinder oriented along the direction $\mathbf{o}$, the dMRI signal due to water diffusion inside the cylinder is approximately $e^{-\left(D^{0} \cos ^{2} \alpha\right) b}$, where $\cos \alpha=\mathbf{g} \cdot \mathbf{o} /(\|\mathbf{g}\|\|\mathbf{o}\|)$, making the theoretical $A D C_{0}^{c y l}=D^{0} \cos ^{2} \alpha, A K_{0}^{c y l}=0$.

We simulated the PGSE sequence: $\delta=2.5 \mathrm{~ms}, \Delta=10 \mathrm{~ms}$. The intrinsic diffusion coefficient was set to $D^{0}=3 \times 10^{-3} \mathrm{~mm}^{2} / \mathrm{s}$ inside the impermeable cylinder. First we placed a straight cylinder of length $50 \mu m$ and radius $R^{c}=1 \mu m$ oriented parallel to the $x$-axis $(\mathbf{o}=[1,0,0])$ and set the gradient direction to be 45 degrees from the $x$-axis: $\mathbf{g} /\|\mathbf{g}\|=[1,1,0] / \sqrt{2}$, making $\alpha=\pi / 4$ and $A D C_{0}^{c y l}=D^{0} / 2=1.5 \times 10^{-3} \mathrm{~mm}^{2} / \mathrm{s}$. We placed water molecules in the center of the cylinder, covering $4 \mu \mathrm{m}$ along the length of the cylinder. The computation box is $C=[-25,25] \mu m \times[-2,2] \mu m \times$ $[-2,2] \mu \mathrm{m}$ and the mesh has spatial discretization $h=0.25 \mu \mathrm{m}$. Next, we rotated the cylinder to $\mathbf{o}=[1,1,0] / \sqrt{2}$ and now measured the diffusion in the direction $\mathbf{g} /\|\mathbf{g}\|=[1,0,0]$. The theoretical $A D C_{0}^{c y l}$ is the same. We use the computation box $C=[-25,25] \mu m \times[-25,25] \mu m \times[-2,2] \mu m$ and three meshes with spatial discretizations $h=0.25, h=0.125, h=0.0625 \mu \mathrm{m}$. We see in in Figure 4(b) that the 
$\log S(b)$ curves are straight lines in $b$ for all the configurations but their slopes differ. This is numerical error associated with the Cartesian discretization of the slanted cylinder so we conducted a convergence study of the $A D C_{0}$ of the "xy-dir" cylinders at four discretization steps: $h=0.25,0.125,0.0625,0.0315 \mu \mathrm{m}$, using the computational domain $C=[-25,25] \mu m \times[-25,25] \mu m \times[-h / 2, h / 2] \mu m$. We computed the $A D C_{0}$ from two b-values: $A D C_{0}=-(\log S(250)-\log S(0)) / 250$. We see in Fig 4(c) that $A D C_{0}$ converges to the exact value $A D C_{0}^{c y l}$ with first order in $h$.

\subsection{Effects of cylindrical cells permeability}

If all the cylinders are thin and straight with their orientations uniformly distributed in a sphere, and are impermeable (as supposed in the model of (Jespersen et al. 2007)), then it is easy to show that:

$$
\begin{aligned}
& S^{c}(b) \approx \frac{\int_{\phi=0}^{\pi} \int_{\theta=0}^{2 \pi} e^{-D^{0} \cos \phi^{2} b} \sin \phi d \theta d \phi}{4 \pi}, \log S^{c}(b)=-\frac{D^{0}}{3} b+\frac{2}{45}\left(D^{0}\right)^{2} b^{2}, \\
& A D C_{0}^{c y l}=\frac{D^{0}}{3}, A K_{0}^{c y l}=\frac{12}{5} .
\end{aligned}
$$

Now, we use an indirect calculation to show that if the cylindrical cells are permeable and the permeable signal is fitted to the no-exchange model of (19), then a first order effect on $v^{c}$ is that $v^{c}$ would be under-estimated, a simulation result that supports the finding of (Jespersen et al. 2010), in the case where the cylinders model dendrites (which are permeable to water). We do this indirectly because of we were not able to do a direct simulation of densely packed randomly oriented non-crossing cylinders embedded in the extra-cellular space, due to the difficult of defining the positions of the non-crossing cylinders, as explained previously.

We suppose that, in the absence of cylindrical cell permeability, the signal is given by (19). We note here that, of course, we have shown in the previous section that the Gaussian diffusion assumption in the extra-cylindrical compartment may not be accurate, but in this section we will just assume the Gaussian diffusion because we want to isolate the effect of the no-exchange assumption.

At relatively low b-values, we can use the following simple approximation of $S^{c}(b)$ :

$$
S^{c}(b)=e^{-A D C_{0}^{c y l} b+\frac{1}{6}\left(A D C_{0}^{c y l}\right)^{2} A K_{0}^{c y l} b^{2}} .
$$

We use (20) rather than the more complicated analytical representation in (Jespersen et al. 2007) because we just want to calculate a leading order change in the estimated $v_{c}$. Thus, it is sufficient to just use two terms in the b-value to approximate $S^{c}(b)$. We fix $A D C_{0}^{c y l}=1.0 \times 10^{-3} \mathrm{~mm}^{2} / \mathrm{s}$ and $A K_{0}^{c y l}=12 / 5$ to be the values that we analytically computed above for straight cylinders with orientations that are uniformly distributed on the unit sphere, where we supposed that the intrinsic $D^{0}=3.0 \times 10^{-3} \mathrm{~mm}^{2} / \mathrm{s}$. In addition, we assume the true volume fraction of the cylindrical cells to be $v^{c}=0.7$ and the effective diffusivity of the combined compartment of the spherical cells and the extra-cellular space to be $D^{e c}=0.3 \times 10^{-3} \mathrm{~mm}^{2} / \mathrm{s}$, as simulated for $v^{s}=0.15, v^{e}=0.15$ previously (see Fig. 2), where we chose the value associated with the $\kappa^{s}=10^{-5} \mathrm{~m} / \mathrm{s}$ and $\Delta=40 \mathrm{~ms}$.

Given $v^{c}, D^{e c}, A D C_{0}^{c y l}, A K_{0}^{c y l}$ defined above, we can now compute the $A D C_{0}$ and $A K_{0}$ of the no-exchange model:

$$
A D C_{0}=\left(1-v^{c}\right) D^{e c}+v^{c} A D C_{0}^{c y l},
$$


$A K_{0}=\frac{3\left(1-v^{c}\right)\left(D^{e c}\right)^{2}+3 v^{c}\left(A D C_{0}^{c y l}\right)^{2}+v^{c}\left(A D C_{0}^{c y l}\right)^{2} A K_{0}^{c y l}-3\left(A D C_{0}\right)^{2}}{\left(A D C_{0}\right)^{2}}$.

Then we create a permeable signal by arguing that at relatively low (but non-negligible) cylindrical cells permeability, the $A D C_{0}$ should remain unchanged, while the $A K_{0}$ should decrease. Thus, we ask the question, if one lowers the $A K_{0}$ of the no-exchange model to $A K_{0}(1-\epsilon)$ to account for cylindrical cells permeability while keeping the $A D C_{0}$ the same, what happens to the estimated $v^{c}$ and $D^{e c}$ if we fix $A D C_{0}^{c y l}$ and $A K_{0}^{c y l}$ at their original values? We note here that of course, it is not possible to write down the complete permeable signal just from knowing the impermeable signal and the permeability coefficient $\kappa$. What we assume about the permeable signal is just that the $A D C_{0}$ stays the same with respect to the impermeable signal and the the $A K_{0}$ is decreased by a certain amount compared to the impermeable signal.

We set the two expressions in (21-22) equal to $\widetilde{A D C_{0}}=A D C_{0}$ and $\widetilde{A K_{0}}=$ $A K_{0}(1-\epsilon)$, and solve for the $\widetilde{v^{c}}$ and $\widetilde{D^{e c}}$ associated with the permeable signal, while keeping the original $A D C_{0}^{c y l}$ and $A K_{0}^{c y l}$. We show the results in Fig 5 for $\epsilon$ from 0 to 50 percent. We see that $\widetilde{v^{c}}$ is under-estimated compared to its true value of 0.7 as $\epsilon$ increases. We also see that $\widetilde{D^{e c}}$ is over-estimated compared to its true value of $D^{e c}=0.3 \times 10^{-3} \mathrm{~mm}^{2} / \mathrm{s}$. This is a simple analysis of one factor that may contribute to the under-estimation of $v^{c}$ due to permeable cylindrical cells observed in (Jespersen et al. 2010). There of course may also be other contributing factors that we did not account for in this analysis.

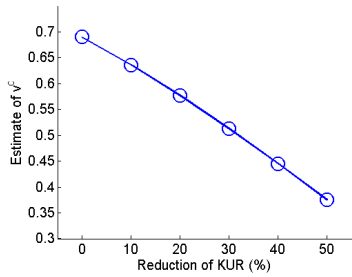

(a) Estimated $v^{c}$

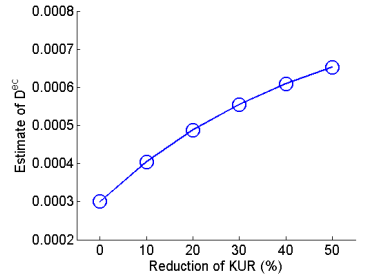

(b) Estimated $D^{e c}$

Figure 5. The results of fitting $\widetilde{A D C_{0}}=A D C_{0}$ and $\widetilde{A K_{0}}=A K_{0}(1-\epsilon)$ of a permeable signal to the no-exchange model in $(19,20)$. As $\epsilon$, the percentage reduction of $A K_{0}$, increases, $\widetilde{v^{c}}$ is more and more under-estimated compared to its true value of 0.7 and $\widetilde{D^{e c}}$ is more and more over-estimated compared to its true value of $D^{e c}=0.3 \times 10^{-3} \mathrm{~mm}^{2} / \mathrm{s}$.

\section{Conclusions}

We presented a method for the numerical solution of the multiple compartments Bloch-Torrey partial differential equation by coupling a standard Cartesian spatial discretization with an adaptive time discretization using the explicit Runge-KuttaChebyshev method and showed that it is more efficient than the Forward Euler time discretization. This method can be easily implemented on multiple processors to shorten the computational time because all interactions between the unknowns are local and it suffices to divide the computational domain into sub-domains that are loaded on the different processors. 
We used this method to simulate the extra-cylindrical compartment dMRI signal in a tissue model of the brain gray matter consisting of cylindrical cells, spherical cells, and the extra-cellular space, and noted that the signal contribution from the extracylindrical compartment may not be Gaussian at some relevant dMRI diffusion times. We also computed that, as a first order effect, neglecting cylindrical cells permeability would result in an under-estimation of the cylindrical cells volume fraction if using a no-exchange model of the diffusion signal attenuation.

\section{Acknowledgment}

This work was funded in part by the Agence Nationale de la Recherche project "SIMUDMRI".

\section{References}

Assaf Y \& Basser P J 2005 'Composite hindered and restricted model of diffusion (CHARMED) MR imaging of the human brain' NeuroImage 27(1), 48-58.

Callaghan P 1997 'A simple matrix formalism for spin echo analysis of restricted diffusion under generalized gradient waveforms' Journal of Magnetic Resonance 129(1), 74-84.

Chabert S, Molko N, Cointepas Y, Le Roux P \& Le Bihan D 2005 'Diffusion tensor imaging of the human optic nerve using a non-CPMG fast spin echo sequence' J. Magn. Reson. Imaging $\mathbf{2 2}(2), 307-310$.

Crank J 1975 The mathematics of diffusion second edn Clarendon Press, Oxford.

Frohlich A F, Ostergaard L \& Kiselev V G 2006 'Effect of impermeable boundaries on diffusionattenuated \{MR\} signal' Journal of Magnetic Resonance 179(2), $223-233$.

Harkins K D, Galons J P, Secomb T W \& Trouard T P 2009 'Assessment of the effects of cellular tissue properties on ADC measurements by numerical simulation of water diffusion' Magn. Reson. Med. 62(6), 1414-1422.

Hasselman D \& Johnson L F 1987 'Effective thermal conductivity of composites with interfacial thermal barrier resistance' Journal of Composite Materials 21(6), 508-515.

Hwang S N, Chin C L, Wehrli F W \& Hackney D B 2003 'An image-based finite difference model for simulating restricted diffusion' Magnetic Resonance in Medicine 50(2), 373-382.

Jensen J H, Helpern J A, Ramani A, Lu H \& Kaczynski K 2005 'Diffusional kurtosis imaging: The quantification of non-Gaussian water diffusion by means of magnetic resonance imaging', Magnetic Resonance in Medicine 53(6), 1432-1440.

Jespersen S N, Bjarkam C R, Nyengaard J R, Chakravarty M M, Hansen B, Vosegaard T, Astergaard L, Yablonskiy D, Nielsen N C \& Vestergaard-Poulsen P 2010 'Neurite density from magnetic resonance diffusion measurements at ultrahigh field: Comparison with light microscopy and electron microscopy' NeuroImage 49(1), 205-216.

Jespersen S N, Kroenke C D, stergaard L, Ackerman J J \& Yablonskiy D A 2007 'Modeling dendrite density from magnetic resonance diffusion measurements' NeuroImage 34(4), 1473-1486.

Latour L L, Svoboda K, Mitra P P \& Sotak C H 1994 'Time-dependent diffusion of water in a biological model system.' Proceedings of the National Academy of Sciences 91(4), 1229-1233.

Le Bihan D, Breton E, Lallemand D, Grenier P, Cabanis E \& Laval-Jeantet M 1986 'MR imaging of intravoxel incoherent motions: application to diffusion and perfusion in neurologic disorders.' Radiology 161(2), 401-407.

Pfeuffer J, Flogel U, Dreher W \& Leibfritz D 1998 'Restricted diffusion and exchange of intracellular water: theoretical modelling and diffusion time dependence of $1 \mathrm{H}$ NMR measurements on perfused glial cells' NMR in Biomedicine 11(1), 19-31.

Price W S, Barzykin A V, Hayamizu K \& Tachiya M 1998 'A model for diffusive transport through a spherical interface probed by pulsed-field gradient NMR' Biophysical Journal 74(5), 22592271.

Russell G, Harkins K D, Secomb T W, Galons J P \& Trouard T P 2012 'A finite difference method with periodic boundary conditions for simulations of diffusion-weighted magnetic resonance experiments in tissue' Physics in Medicine and Biology 57(4), N35.

Sen P N \& Basser P J 2005 'A model for diffusion in white matter in the brain' Biophys J 89(5), 29272938. 
Sommeijer B P, Shampine L F \& Verwer J G 1998 'RKC: An explicit solver for parabolic PDEs' Journal of Computational and Applied Mathematics 88(2), 315-326.

Stejskal E O \& Tanner J E 1965 'Spin diffusion measurements: Spin echoes in the presence of a time-dependent field gradient' The Journal of Chemical Physics 42(1), 288-292.

Sukstanskii A L \& Yablonskiy D A 2002 'Effects of restricted diffusion on MR signal formation' Journal of Magnetic Resonance 157(1), 92-105.

Szafer A, Zhong J \& Gore J C 1995 'Theoretical model for water diffusion in tissues' Magn. Reson. Med. 33(5), 697-712.

Torquato S \& Rintoul M D 1995 'Effect of the interface on the properties of composite media' Phys. Rev. Lett. 75(22), 4067-.

Torrey H 1956 'Bloch equations with diffusion terms' Physical Review Online Archive (Prola) 104(3), 563-565.

Xu J, Does M \& Gore J 2007 'Numerical study of water diffusion in biological tissues using an improved finite difference method.' Physics in medicine and biology $\mathbf{5 2}(7)$. 